\title{
Characterization and Optimization of L-Malic Acid ๑ Production by Some Clinical Isolates of Aureobasidium pullulans
}

\author{
Taha Jafarian-Haris $^{1}$, Alireza Tavakkoli ${ }^{2}$ (D), Mohammad Javad Najafzadeh ${ }^{3}$ (D, Abolghasem Danesh ${ }^{1}$ (D) \\ 1. Biotechnology Research Center, Pharmaceutical Technology Institute, Mashhad University of Medical Sciences, Mashhad, Iran. \\ 2. Department of Pharmacognosy, School of Pharmacy, Mashhad University of Medical Sciences, Mashhad, Iran. \\ 3. Department of Parasitology and Mycology, Faculty of Medicine, Mashhad University of Medical Sciences, Mashhad, Iran.
}

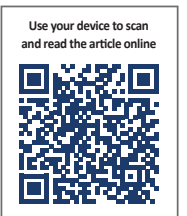

Citation Jafarian-Haris T, Tavakkoli AR, Najafzadeh MJ, Danesh A. Characterization and Optimization of L-Malic Acid Production by Some Clinical Isolates of Aureobasidium pullulans. Research in Molecular Medicine. 2020; 8(4):209-214. https:// doi.org/10.32598/rmm.8.4.1173.1

doi https://doi.org/10.32598/rmm.8.4.1173.1

Keywords:

Poly L-malic acid, L-Malic acid, Aureobasidium pullulans, Fungal natural products

\begin{abstract}
A B S T RA C T
Background: Poly-L-malic acid (PLMA) comprises aliphatic polyester polymers with broad applications in pharmaceutical industries. The fungal microorganisms are among the best natural sources recruited to supply L-malic acid (MA) as a precursor of PLMA. In this study, we investigated MA production ability of 7 clinical isolated of the fungus Aureobasidium pullulans.

Materials and Methods: Seven clinical isolates of $A$. pullulans acquired from Westerdijk Fungal Biodiversity Institute were studied, and the isolate with the highest total MA production was selected for the optimization process. We tried to optimize the output by applying different concentrations of $\mathrm{CaCO} 3$ in fungus medium $(1.5 \%, 3 \%$, and $6 \%)$ and various incubation temperatures $\left(27^{\circ} \mathrm{C}, 32^{\circ} \mathrm{C}\right.$, and $37^{\circ} \mathrm{C}$ ) during 3,7 , and 14 days.

Results: Intra-strains variation was significantly strong ( $\mathrm{P}<0.0001)$, and the highest production of MA was carried out by the isolate A. pullulans var. melanigenum dH 21931, UTHSC 06-456. The amount of MA produced by this strain was significantly higher in medium with $3 \% \mathrm{CaCO}_{3}$ compared with other concentrations of $\mathrm{CaCO}_{3}$ and after 7 days incubation than the other fermentation times $(\mathrm{P}<0.05)$. Although MA production was higher at $27^{\circ} \mathrm{C}$, the differences between the investigated various temperatures were not significant $(\mathrm{P}>0.05)$.
\end{abstract}

Conclusion: Overall, we obtained the highest MA production in Sabouraud dextrose agar (SDA) medium with $3 \% \mathrm{CaCO}_{3}$ at $27^{\circ} \mathrm{C}$ after 7 days of incubation. Our study indicated that the fermentation period and $\mathrm{CaCO}_{3}$ concentration significantly alter MA production in A. pullulans var. melanigenum.

\section{Introduction}

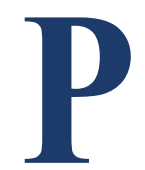

oly L-malic acid (PLMA) consists of repetitive L-malic acid (MA) units, a four-carbon dicarboxylic acid unit polymers that are widely used as the second-generation of biomaterials for drug delivery systems [1]. Depending on the connection of their MA units, these polymers have three forms of poly- $\alpha$, poly- $\beta$, and poly- $\alpha, \beta$, each of which could be synthesized chemically or obtained from living organisms. The $\beta$-type has the broadest applications in drug-carrier complexes because of its versatile pen-

\section{* Corresponding Author:}

Abolghasem Danesh, PhD.

Address: Biotechnology Research Center, Pharmaceutical Technology Institute, Mashhad University of Medical Sciences, Mashhad, Iran.

Phone: +98 (51) 31801202

E-mail:danesha@mums.ac.ir 
dant $\alpha$-carboxyl groups, which provide sites for chemical modifications to target the delivery of drugs [2]. PLMA could also be easily ionized in water mediums and provide highly soluble poly-carboxylates [3]. In this condition, it can conjugate with cationic compounds such as anticancer drug doxorubicin during a stable ionic reaction. Additionally, these carboxylic polymers are suitable candidates for $\mathrm{pH}$-sensitive drug delivery systems [4].

At present, the main production method of MA as a precursor of PLMA is the hydration of fumaric acid or maleic acid under high pressure and temperature or fumarate biotransformation by fumarase enzyme. However, these processes require the malic anhydride-derived from petroleum- or fumaric acid and relatively expensive catalysts and therefore are limited due to economic issues [5-7]. Therefore, recent research is focused on developing microbial sources for MA production by using renewable raw materials. Several different microorganisms are studied for this purpose. Some of them such as Zygosaccharomyces rouxii, Brevibacterium flavum, Saccharomyces cerevisiae, and different Aspergillus spp. as well as some metabolically engineered Escherichia coli can produce MA from glucose or other carbohydrates [9-14].

A. pullulans is a melanized yeast-like saprophytic fungus, which is widely found in the environment and can cause rare infections such as fungemia and phaeohyphomycosis in immunodeficient and critically-ill patients $[15,16]$. This fungus is currently applied for commercial production of a food additive namely pullulan which is a polymer of maltotriose. It is also used to bio-remediate some environmental pollutions, such as oil spillage pollutions [17]. Some studies reported different antifungal and anti-bacterial metabolites from this fungus $[18,19]$.Moreover, some other research studies have shown that $A$. pullulans can encode some valuable enzymes such as alkaline protease [20] and endo-1,4- $\beta$-xylanase [21].

Additionally, several studies have indicated that A. pullulans could be considered a good potential source for PLMA supply [22-24]. The question is if opportunistic pathogenic isolates of $A$. pullulans can produce MA and what nutritional elements can alter their MA production. In this study, we investigated the ability of some clinical isolates of Aureobasidium for MA production. We tried to optimize production and increase efficiency by altering some environmental and nutritional factors.

\section{Methods}

\section{Chemicals}

Sabouraud dextrose agar (SDA) medium, glucose, $\mathrm{CaCO}_{3}$, $\mathrm{KH}_{2} \mathrm{PO}_{4}, \mathrm{KCl}, \mathrm{NaNO}_{3}, \mathrm{MgSO}_{4} \cdot 7 \mathrm{H}_{2} \mathrm{O}$, and sulfuric acid were purchased from Merck, Germany. L-malic acid assay kit (Manual Format) was obtained from Megazyme, Ireland.

\section{Fungal samples preparation}

At first, a collection of 40 fungal isolates of Aureobasidium spp. were received from Prof G. Sybren de Hoog collection in Westerdijk Fungal Biodiversity Institute, the Netherland (presented by $\mathrm{dH}$ codes). Each of the 40 types of fungi was inoculated into two tubes containing the SDA medium. The samples were then incubated at $25^{\circ} \mathrm{C}$ for 4 days, and among them, only 7 isolates were recovered (Table 1). Next, each of these 7 isolates was inoculated to three $250-\mathrm{mL}$ Erlenmeyer flasks (in addition to three ones without any inoculation for the negative standard), containing a liquid medium consisting of glucose (12 g), $\mathrm{CaCO}_{3}(3 \mathrm{~g}), \mathrm{NaNO} 3$ (0.2 g), $\mathrm{KH}_{2} \mathrm{PO}_{4}(0.01 \mathrm{~g}), \mathrm{KCl}(0.05 \mathrm{~g})$ and $\mathrm{MgSO}_{4} \cdot 7 \mathrm{H}_{2} \mathrm{O}(0.02 \mathrm{~g})$ in $100 \mathrm{~mL}$ distilled water. $\mathrm{CaCO}_{3}$ solution was prepared and autoclaved separately due to the possible reaction with other medium components in high temperatures. All flasks were sterilized by autoclaving at $121^{\circ} \mathrm{C}$ and incubated in a shaker incubator for 7 days at $27^{\circ} \mathrm{C}$. The fermented mediums were then transferred to Falcon tubes and centrifuged (8000 rpm for 30 minutes), and the resulted supernatants of each isolate were transferred equally to test tubes. The tubes were treated with the same volume of sulfuric acid (2 M) and incubated at $100^{\circ} \mathrm{C}$ for 12 hours to hydrolyze PLMA into MA. The prepared test samples were examined by L-malic acid assay kit based on their UV absorbance at $340 \mathrm{~nm}$. The final concentration of MA in fermented mediums was calculated and reported as $\mathrm{g} / \mathrm{L}$.

\section{Optimization procedure}

Based on the previous step's results, the isolate dH 21931, UTHSC 06-456, which produces the highest amount of MA, was selected for the optimization process (Figure 1). First, the selected isolate was fermented as per the previously-described method except for the difference in medium $\mathrm{CaCO}_{3}$ concentration, which was adjusted as different states of $1.5 \%$, $3 \%$, and $6 \%$ to find in which condition the highest production of MA occurs. Next, the best $\mathrm{CaCO}_{3}$ concentration was fixed, and the flasks were incubated at different temperatures $\left(27^{\circ} \mathrm{C}, 32^{\circ} \mathrm{C}\right.$, or $37^{\circ} \mathrm{C}$ ) for different periods (3 days, 7 days, or 14 days). Each of the specific planned conditions of fermentation (including temperature, time, and $\mathrm{CaCO}_{3}$ concentration) was carried out in three separate flasks, and reported values were the average of their results.

\section{Statistical analysis}

GraphPad Prism software v. 8.3.0 for Windows (GraphPad Software, La Jolla California USA) was used for statistical 


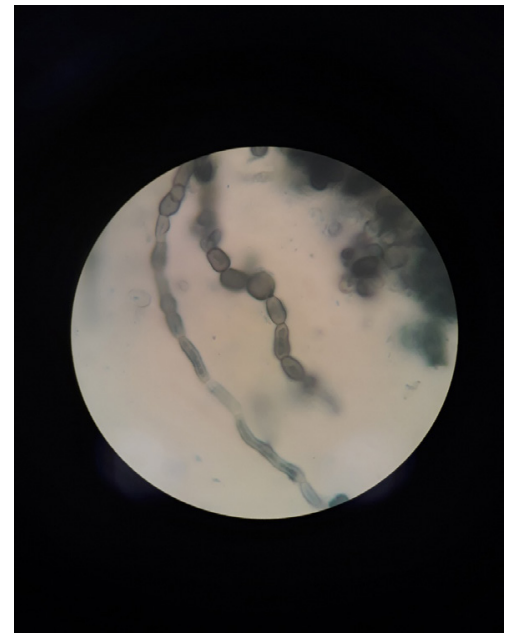

grmm

Figure 1. Melanized conidiophores (right) and short hypha (left) of A. pullulans var. melanigenum dH 21931, UTHSC 06456 on Sabouraud dextrose agar medium $\times 1000$

analysis. The results were presented as Mean \pm SD. The Shapiro-Wilk pretest was used to confirm the normal statistical distribution in each group. One-way or 2-way ANOVA and Tukey-Kramer posttest were used to compare the different groups. $\mathrm{P}<0.05$ was considered statistically significant.

\section{Results}

\section{Screening of the isolates}

As shown in Figure 2, the highest MA production was obtained by the isolate dH 21931, UTHSC 06-456. The intra-isolate variations were strongly significant $(\mathrm{P}<0.0001)$, as the highest strain production was 8 -fold more than the lowest one.

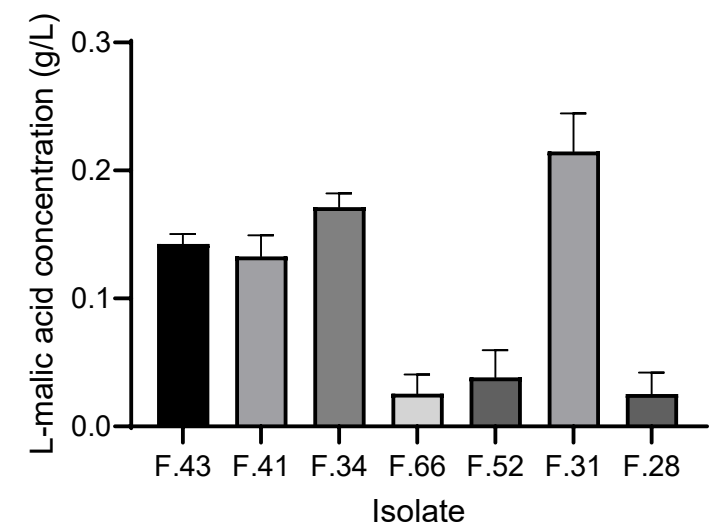

grmm

Figure 2. MA concentration in fermented medium of seven selected fungal isolates, after 7 days of incubation at $27^{\circ} \mathrm{C}$

The results are shown as Mean $\pm S D(n=3)$.

\section{Optimization results}

Figure 3 shows the average amount of MA produced by isolate $\mathrm{dH}$ 21931, UTHSC 06-456 in different concentrations of $\mathrm{CaCO}_{3}$. The results showed that the highest amount of MA production happened with $3 \% \mathrm{CaCO}_{3}$ to the culture medium, and subsequently $6 \%$ and $1.5 \%$. Statistical analysis with 1-way ANOVA showed a significant difference between these three concentrations in the production of MA $(\mathrm{P}=0.0413)$. Tukey-Kramer posttest indicated that in multiple comparisons, the significant difference is only observed in the case of $1.5 \%$ vs. $3 \%$ concentrations.

Table 2 presents the amount of MA produced by $\mathrm{dH} 21931$, UTHSC 06-456 isolate on 3, 7, and 14 days after inoculation, at different temperatures $\left(27^{\circ} \mathrm{C}, 32^{\circ} \mathrm{C}\right.$ or $\left.37^{\circ} \mathrm{C}\right)$. As indicated in Figure 4, the best output was achieved after 7 days at $27^{\circ} \mathrm{C}$. Statistical analysis by 2-way ANOVA test showed no significant difference between distinct incubation temperatures $(\mathrm{P}>0.05)$. Still, the fermentation period had a significant effect on MA amount in general ( $\mathrm{P}=0.0288)$. The TukeyKramer postest indicated that in multiple comparisons, a significant difference is observed in the case of 3 days vs. 7 days $(\mathrm{P}=0.0226)$. This correlation was stronger in the case of $27^{\circ} \mathrm{C}$ temperature, and 7 days yield was significantly higher than 3 days $(\mathrm{P}=0.0115)$.

\section{Discussion}

Fungal microorganisms are among the notable sources of green production of MA $[25,26]$. Considering some of the previous studies, which had highlighted the effect of incubation temperature and $\mathrm{CaCO}_{3}$ concentration in MA production by fungal microorganisms such as Zygosaccharomyces

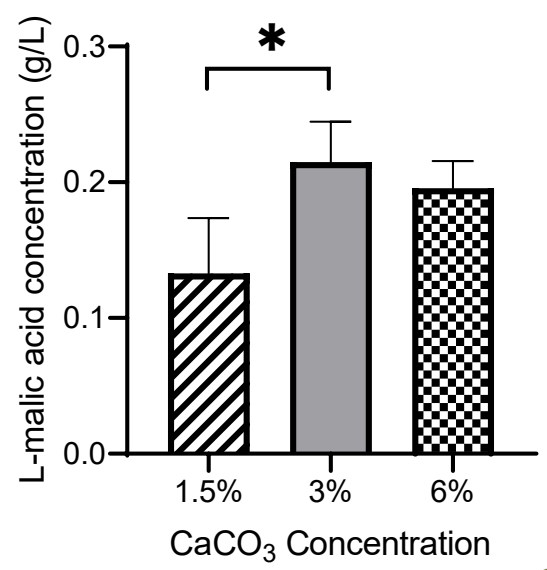

कrmm

Figure 3. MA concertation in $\mathrm{dH} 21931$, UTHSC 06-456 isolate medium with different concentrations of $\mathrm{CaCO} 3$ at $27^{\circ} \mathrm{C}$ after 7 days of incubation

Results are shown as Mean \pm SD $(n=3)$. * Indicates $P<0.05$ and different significance. 
Table 1. Characteristics of seven investigated isolates

\begin{tabular}{|c|c|c|c|c|}
\hline No. & Identification & Code & Source & Country \\
\hline F.43 & A. pullulans var. pullulans & dH 21943, UTHSC 07-2037 & Cerebrospinal fluid & The USA \\
\hline F.41 & A. pullulans var. melanigenum & dH 21941, UTHSC 07-1927 & Bronchial wash & The USA \\
\hline F.34 & A. pullulans var. melanigenum & dH 21934, UTHSC 07-22 & Ethmoid sinus & The USA \\
\hline F.66 & A. pullulans var. melanigenum & dH 21966, UTHSC 10-1003 & Bronchial wash & The USA \\
\hline F.52 & A. pullulans var. pullulans & dH 21952, UTHSC 08-1356 & Toe nail & The USA \\
\hline F.31 & A. pullulans var. melanigenum & dH 21931, UTHSC 06-456 & Forehead & The USA \\
\hline F.28 & A. pullulans var. melanigenum & dH 21928, UTHSC 03-3450 & Bone marrow & The USA \\
\hline
\end{tabular}

rouxii and Schizophyllum commune [26], we decided to investigate these factors effects in the case of $A$. pullulans var. melanigenum.

$\mathrm{CaCO}_{3}$ addition into the fermentation medium is performed for two major purposes: to provide $\mathrm{CO} 2$ for the reductive Tricarboxylic Acid Cycle (TCA) pathway, where pyruvate is converted to oxaloacetate and then malate, and buffer the $\mathrm{pH}$ at $\sim 6.0$ [27]. It has been well documented that the con- centration of $\mathrm{CaCO}_{3}$ in the medium is contributed to PLMA production by $A$. pullulans, but the reports are controversial. Zhang et al. (2011), for example, reported $4 \% \mathrm{CaCO}_{3}$ as the optimum $\mathrm{CaCO}_{3}$ concentration for PLMA production by $A$. pullulans [10], while another research reported that a medium containing $6.5 \%$ of $\mathrm{CaCO}_{3}$ is ideal [28]. On the other hand, $\mathrm{Cao}$ et al. has obtained the highest PLMA production by adding $1.5 \% \mathrm{CaCO}_{3}$ to $A$. pullulans medium [29]. The present study proved a significant correlation between

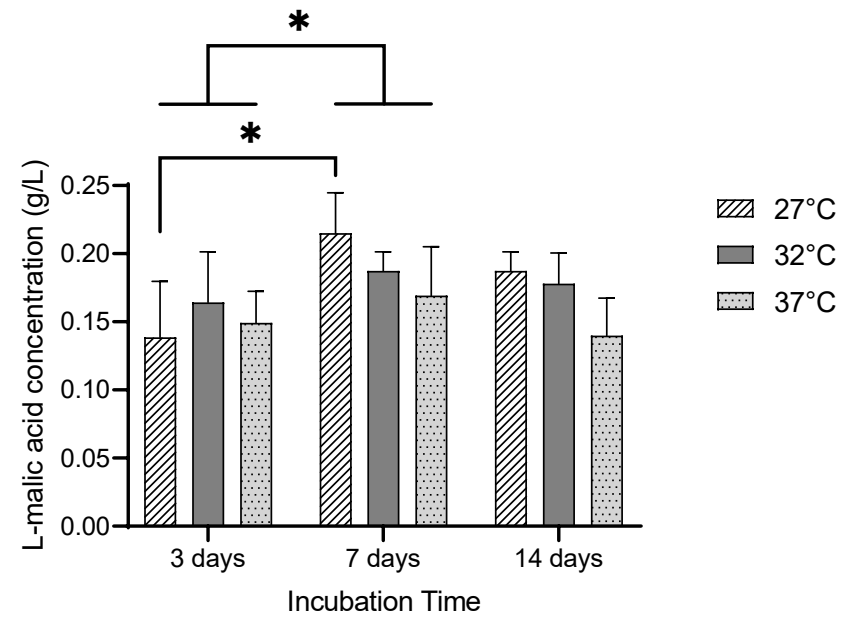

grmm

Figure 4. MA concertation in dH 21931, UTHSC 06-456 isolate medium with different fermentation temperatures and times The results are presented as Mean \pm SD $(n=3)$. *Indicates $\mathrm{P}<0.05$ and different significance.

Table 2. MA concertation in dH 21931, UTHSC 06-456 isolate medium with different fermentation temperatures and times

\begin{tabular}{cccc}
\hline Incubation Time & $\mathbf{2 7}{ }^{\circ} \mathrm{C}$ & $\mathbf{3 2}{ }^{\circ} \mathrm{C}$ & $37^{\circ} \mathrm{C}$ \\
\hline 3 days & $0.138 \pm 0.041 \mathrm{~g} / \mathrm{L}$ & $0.164 \pm 0.037 \mathrm{~g} / \mathrm{L}$ & $0.149 \pm 0.023 \mathrm{~g} / \mathrm{L}$ \\
7 days & $0.215 \pm 0.029 \mathrm{~g} / \mathrm{L}$ & $0.187 \pm 0.014 \mathrm{~g} / \mathrm{L}$ & $0.169 \pm 0.035 \mathrm{~g} / \mathrm{L}$ \\
14 days & $0.187 \pm 0.014 \mathrm{~g} / \mathrm{L}$ & $0.178 \pm 0.022 \mathrm{~g} / \mathrm{L}$ & $0.140 \pm 0.027 \mathrm{~g} / \mathrm{L}$ \\
\hline
\end{tabular}


$\mathrm{CaCO}_{3}$ concentration and MA production by A. pullulans; although, we achieved the highest MA production $(0.21 \pm 0.02$ $\mathrm{g} / \mathrm{L}$ ) by adding $3 \% \mathrm{CaCO}_{3}$ in $\mathrm{A}$. pullulans fermentation medium. The observed differences in the $\mathrm{CaCO}_{3}$ concentration effect may be contributed to intra-strains variations; however, further investigations are needed.

Besides, our findings represented that MA concentration was highest after 7 days of fermentation and then decreased to the $14^{\text {th }}$ day. We measured total MA in our samples by treating them with sulfuric acid ( $2 \mathrm{M}$ ) before assay by kit, which hydrolyzes the present PLMA into MA [26]. It is shown that in fungal cells, the production of PLMA as a major form of MA is associated with cell growth in the early exponential growth phase [27, 30], so the end of exponential growth in the second week of fermentation could be the cause of the observed MA level decrease.

\section{Conclusion}

MA production ability significantly differs between clinical isolates of $A$. pullulans. By studying the role of medium $\mathrm{CaCO}_{3}$ concentration and incubation time and temperature on MA production on the isolate $\mathrm{dH}$ 21931, UTHSC 06-456 of A. pullulans var. melanigenum, we found that the output is significantly affected by medium $\mathrm{CaCO}_{3}$ concentration and fermentation period. Overall, the highest amount of MA production was obtained after 7 days of incubation at $27^{\circ} \mathrm{C}$, with a medium contained $3 \% \mathrm{CaCO}_{3}$. The limitations of our study include the low scale of fermentation and missing some other potentially useful factors on MA biosynthesis yield, such as $\mathrm{pH}$ and trace elements of the medium. Besides, we propose to perform additional studies on other Aureobasidium spp. and genetic studies on the MA biosynthesis pathway.

\section{Ethical Considerations}

\section{Compliance with ethical guidelines}

All ethical principles are considered in this article. The participants were informed of the purpose of the research and its implementation stages. They were also assured about the confidentiality of their information and were free to leave the study whenever they wished, and if desired, the research results would be available to them.

\section{Funding}

This work was supported by Mashhad University of Medical Sciences (Grant No.: 921255).

\section{Authors' contribution's}

Conceptualization and Supervision: Abolghasem Danesh; Methodology: Mohammad Javad Najafzadeh, Abolghasem Danesh; Investigation, Writing - original draft, and Writing review \& editing: All authors; Data collection: Taha JafarianHaris; Data analysis: Taha Jafarian-Haris, Alireza Tavakkoli; Funding acquisition and Resources: Mohammad Javad Najafzadeh, Abolghasem Danesh.

\section{Conflict of interest}

The authors declared no conflict of interest.

Acknowledgements

This work was supported by Mashhad University of Medical Sciences (Grant No.: 921255).

\section{References}

[1] Manitchotpisit P, Skory CD, Peterson SW, Price NP, Vermillion KE, Leathers TD. Poly(beta-L-malic acid) production by diverse phylogenetic clades of Aureobasidium pullulans. J Ind Microbiol Biotechnol. 2012; 39(1):125-32. [DOI:10.1007/s10295-011-1007-7] [PMID]

[2] Chi Z, Liu GL, Liu CG, Chi ZM. Poly(beta-L-malic acid) (PMLA) from Aureobasidium spp. and its current proceedings. Appl Microbiol Biotechnol. 2016; 100(9):3841-51. [DOI:10.1007/s00253016-7404-0] [PMID]

[3] Zhang H, Cai J, Dong J, Zhang D, Huang L, Xu Z, et al. Highlevel production of poly (beta-L-malic acid) with a new isolated Aureobasidium pullulans strain. Appl Microbiol Biotechnol. 2011; 92(2):295-303. [DOI:10.1007/s00253-011-3358-4] [PMID]

[4] Lanz-Landázuri A, Martínez de Ilarduya A, García-Alvarez M, Muñoz-Guerra S. Poly( $\beta$,L-malic acid)/Doxorubicin ionic complex: A pH-dependent delivery system. React Funct Polym. 2014; 81:45-53. [DOI:10.1016/j.reactfunctpolym.2014.04.005]

[5] Bressler E, Pines O, Goldberg I, Braun S. Conversion of fumaric acid to L-malic by sol-gel immobilized Saccharomyces cerevisiae in a supported liquid membrane bioreactor. Biotechnol Prog. 2002; 18(3):445-50. [DOI:10.1021/bp010139t] [PMID]

[6] Stojkovič G, Plazl I, Žnidaršič-Plazl P. L-Malic acid production within a microreactor with surface immobilised fumarase. Microfluid Nanofluidics. 2011; 10(3):627-35. [DOI:10.1007/s10404010-0696-y]

[7] Vrsalović Presečki A, Zelić B, Vasić-Rački Đ. Modelling of continuous L-malic acid production by porcine heart fumarase and fumarase in yeast cells. Chem Biochem Eng Q. 2009; 23(4):51925. https://hrcak.srce.hr/45416

[8] Zheng H, Ohno Y, Nakamori T, Suye S. Production of L-malic acid with fixation of HCO3(-) by malic enzyme-catalyzed reaction based on regeneration of coenzyme on electrode modified 
by layer-by-layer self-assembly method. J Biosci Bioeng. 2009; 107(1):16-20. [DOI:10.1016/j.jbiosc.2008.09.009] [PMID]

[9] Chibata I, Tosa T, Takata I. Continuous production of L-malic acid by immobilized cells. Trends Biotechnol. 1983; 1(1):9-11. [DOI:10.1016/0167-7799(83)90019-7]

[10] Zhang X, Wang X, Shanmugam KT, Ingram LO. L-malate production by metabolically engineered Escherichia coli. Appl Environ Microbiol. 2011; 77(2):427-34. [DOI:10.1128/AEM.01971-10] [PMID] [PMCID]

[11] Zelle RM, de Hulster E, van Winden WA, de Waard P, Dijkema C, Winkler AA, et al. Malic acid production by Saccharomyces cerevisiae: Engineering of pyruvate carboxylation, oxaloacetate reduction, and malate export. Appl Environ Microbiol. 2008; 74(9):2766-77. [DOI:10.1128/AEM.02591-07] [PMID] [PMCID]

[12] West TP. Malic acid production from thin stillage by Aspergillus species. Biotechnol Lett. 2011; 33(12):2463-7. [DOI:10.1007/ s10529-011-0720-7] [PMID]

[13] Moon SY, Hong SH, Kim TY, Lee SY. Metabolic engineering of Escherichia coli for the production of malic acid. Biochem Eng J. 2008; 40(2):312-20. [DOI:10.1016/j.bej.2008.01.001]

[14] Taing O, Taing K. Production of malic and succinic acids by sugar-tolerant yeast Zygosaccharomyces rouxii. Eur Food Res Technol. 2007; 224(3):343-7. [DOI:10.1007/s00217-006-0323-z]

[15] Mershon-Shier KL, Deville JG, Delair S, Fothergill AW, Wickes B, de Hoog GS, et al. Aureobasidium pullulans var. melanigenum fungemia in a pediatric patient. Med Mycol. 2011; 49(1):80-3. [DO I:10.3109/13693786.2010.490925] [PMID]

[16] Najafzadeh MJ, Sutton DA, Keisari MS, Zarrinfar H, De Hoog GS, Chowdhary A, et al. In vitro activities of eight antifungal drugs against 104 environmental and clinical isolates of $\mathrm{Au}$ reobasidium pullulans. Antimicrob Agents Chemother. 2014; 58(9):5629-31. [DOI:10.1128/AAC.03095-14] [PMID] [PMCID]

[17] Deshpande MS, Rale VB, Lynch JM. Aureobasidium pullulans in applied microbiology: A status report. Enzyme Microb Technol. 1992; 14(7):514-27. [DOI:10.1016/0141-0229(92)90122-5]

[18] Endo M, Takesako K, Kato I, Yamaguchi H. Fungicidal action of aureobasidin A, a cyclic depsipeptide antifungal antibiotic, against Saccharomyces cerevisiae. Antimicrob Agents Chemother. 1997; 41(3):672-6. [DOI:10.1128/AAC.41.3.672] [PMID] [PMCID]

[19] NE Kalantar, RL Deopurkar, Kapadnis B. Antistaphylococcal metabolite from Aureobasidium pullulans: Production and characterization. Afr J Cli and Exp Microbiol. 2005; 6(3):177-87. [DOI:10.4314/ajcem.v6i3.7419]

[20] Chi Z, Ma C, Wang P, Li HF. Optimization of medium and cultivation conditions for alkaline protease production by the marine yeast Aureobasidium pullulans. Bioresour Technol. 2007; 98(3):534-8. [DOI:10.1016/j.biortech.2006.02.006] [PMID]

[21] Ohta K, Moriyama S, Tanaka H, Shige T, Akimoto H. Purification and characterization of an acidophilic xylanase from Aureobasidium pullulans var. melanigenum and sequence analysis of the encoding gene. J Biosci Bioeng. 2001; 92(3):262-70 [DOI:10.1016/S1389-1723(01)80260-7]

[22] Cao W, Luo J, Qi B, Zhao J, Qiao C, Ding L-H, et al. beta-poly(Lmalic acid) production by fed-batch culture of Aureobasidium pullulans ipe-1 with mixed sugars. Eng Life Sci. 2014; 14(2):180-9. [DOI:10.1002/elsc.201200189]
[23] Cao W, Wang Y, Shen F, Luo J, Yin J, Qiao C, et al. Efficient $\beta$-poly(L-malic acid) production from Jerusalem artichoke by Aureobasidium pullulans ipe-1 immobilized in luffa sponge matrices. Bioresour Technol. 2019; 288:121497. [DOI:10.1016/j.bii ortech.2019.121497] [PMID]

[24] Cao W, Cao W, Shen F, Luo J, Yin J, Qiao C, et al. Membraneassisted $\beta$-poly(L-malic acid) production from bagasse hydrolysates by Aureobasidium pullulans ipe-1. Bioresour Technol. 2020; 295:122260. [DOI:10.1016/j.biortech.2019.122260] [PMID]

[25] Chi Z, Wang ZP, Wang GY, Khan I, Chi ZM. Microbial biosynthesis and secretion of L-malic acid and its applications. Crit Rev Biotechnol. 2016; 36(1):99-107. [DOI:10.3109/07388551.2014.9244 74] [PMID]

[26] West T. Microbial production of malic acid from biofuel-related coproducts and biomass. Ferment. 2017; 3:14. [DOI:10.3390/ferr mentation3020014]

[27] Zou X, Cheng C, Feng J, Song X, Lin M, Yang ST. Biosynthesis of polymalic acid in fermentation: Advances and prospects for industrial application. Crit Rev Biotechnol. 2019;39(3):408-21. [D OI:10.1080/07388551.2019.1571008] [PMID]

[28] Wang YK, Chi Z, Zhou HX, Liu GL, Chi ZM. Enhanced production of $\mathrm{Ca}(2)(+)$-polymalate (PMA) with high molecular mass by Aureobasidium pullulans var. pullulans MCW. Microb Cell Fact. 2015; 14:115. [DOI:10.1186/s12934-015-0296-3] [PMID] [PMCID]

[29] Cao W, Chen X, Luo J, Yin J, Qiao C, Wan Y. High molecular weight beta-poly(L-malic acid) produced by A. pullulans with $\mathrm{Ca}(2)(+)$ added repeated batch culture. Int J Biol Macromol. 2016; 85:192-9. [DOI:10.1016/j.ijbiomac.2015.12.056] [PMID]

[30] Cao W, Luo J, Zhao J, Qiao C, Ding L, Qi B, et al. Intensification of $\beta$-poly(L: -malic acid) production by Aureobasidium pullulans ipe-1 in the late exponential growth phase. J Ind Microbiol Biotechnol. 2012; 39(7):1073-80. [DOI:10.1007/s10295-012-1111-3] [PMID] 